\title{
Essential list of medicinal products for rare diseases: recommendations from the IRDiRC Rare Disease Treatment Access Working Group
}

\author{
William A. Gahl' ${ }^{1}$, Durhane Wong-Rieger ${ }^{2 *}$ (D) Virginie Hivert ${ }^{3}$, Rachel Yang ${ }^{4}$, Galliano Zanello ${ }^{5}$ and \\ Stephen Groft ${ }^{6}$
}

\begin{abstract}
Background: Treatments are often unavailable for rare disease patients, especially in low-and-middle-income countries. Reasons for this include lack of financial support for therapies and onerous regulatory requirements for approval of drugs. Other barriers include lack of reimbursement, administrative infrastructure, and knowledge about diagnosis and drug treatment options. The International Rare Diseases Research Consortium set up the Rare Disease Treatment Access Working Group with the first objective to develop an essential list of medicinal products for rare diseases.

Results: The Working Group extracted 204 drugs for rare diseases in the FDA, EMA databases and/or China's NMPA databases with approval and/or marketing authorization. The drugs were organized in seven disease categories: metabolic, neurologic, hematologic, anti-inflammatory, endocrine, pulmonary, and immunologic, plus a miscellaneous category.

Conclusions: The proposed list of essential medicinal products for rare diseases is intended to initiate discussion and collaboration among patient advocacy groups, health care providers, industry and government agencies to enhance access to appropriate medicines for all rare disease patients throughout the world.
\end{abstract}

\section{Introduction}

A significant unmet need for individuals living with rare diseases is access to beneficial therapies, even those that are approved by major regulatory bodies and are considered as standards of care by experts throughout the world. This issue is especially apparent in low-and-middle-income countries (LMICs) [1] but also affects a substantial proportion of eligible patients in high-income jurisdictions. Of course, this inequity in access applies not only to rare disease drugs but also therapies for common, chronic diseases [2]. However, the disparity is even greater for rare disease treatments [3]. Moreover, while there are international initiatives and programs to make

\footnotetext{
*Correspondence: durhane@sympatico.ca

${ }^{2}$ Canadian Organization for Rare Disorders, Toronto, Canada

Full list of author information is available at the end of the article
}

available therapies for conditions affecting large patient populations, such as diabetes, HIV and cancer, there has been little action to improve access to drugs for those suffering from rare conditions [4].

To stimulate a broad response to this unmet need, the International Rare Diseases Research Consortium (IRDiRC) established the Rare Disease Treatment Access Working Group (RDTAWG) with three aims: (1) To improve standards of care for RD patients by promoting access to approved medicines; (2) To initiate research into the barriers to accessing RD drugs, especially in LMICs; and (3) To define opportunities to address those barriers.

This paper is the first of a three-part series with special focus on lack of access to orphan and rare disease drugs in LMICs and also inequitable access in highincome countries. This first paper presents a curated list

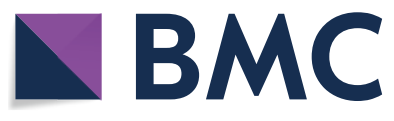

(c) The Author(s) 2021. Open Access This article is licensed under a Creative Commons Attribution 4.0 International License, which permits use, sharing, adaptation, distribution and reproduction in any medium or format, as long as you give appropriate credit to the original author(s) and the source, provide a link to the Creative Commons licence, and indicate if changes were made. The images or other third party material in this article are included in the article's Creative Commons licence, unless indicated otherwise in a credit line to the material. If material is not included in the article's Creative Commons licence and your intended use is not permitted by statutory regulation or exceeds the permitted use, you will need to obtain permission directly from the copyright holder. To view a copy of this licence, visit http://creativecommons.org/licenses/by/4.0/. The Creative Commons Public Domain Dedication waiver (http://creativeco mmons.org/publicdomain/zero/1.0/) applies to the data made available in this article, unless otherwise stated in a credit line to the data. 
of medicines considered to be essential for rare disorders and already approved by the US Food and Drug Administration (FDA), the European Medicines Agency (EMA), and/or China's National Medical Products Administration (NMPA). The second paper will discuss the barriers to access stratified by types of therapy, characteristics of rare disease populations, and key country parameters such as investment in health, health system capabilities, and rare disease priorities. That paper will also review some existing mechanisms for providing therapeutic access for rare and non-rare conditions. The third paper will consider strategies for improving access directed toward barriers identified along the patient pathway, in general and specific to rare conditions.

\section{Methods}

The IRDiRC RDTAWG developed a list of essential medicinal products for rare conditions; the list was not intended to include all medicines used to treat rare diseases but those that could be considered as essential based on approvals by key regulatory agencies in the USA, the European Union (EU) and China for the treatment of rare conditions. Two approaches were used to compile the list. The first approach was to start with databases of medicinal products with designated orphan status or marketing authorizations for rare disease indications. The initial references were the USA FDA Orphan Drug Product Designation database for products approved in the USA [5], the Orphanet list of medicinal products for rare diseases in Europe (2020) [6], and the EMA database of approved products and designations [7]. All drugs with orphan designations and FDA approval were extracted and a list was created, arranged by rare condition usage, generic (medicinal) name, and regulatory approval status. Medicinal products for rare diseases that have European Union marketing authorizations (with or without orphan drug designation) were then collated by using the Orphanet and EMA databases. To round out the list, China's first published Rare Diseases Catalog [8,9] of 121 rare diseases/disease types and China's Lists of Novel Drugs Approved in Other Jurisdictions with Urgent Clinical Needs [10-12] were consulted to develop a list of medicines that were approved for the treatment of recognized rare conditions.

The second approach to developing the essential rare disease medicines list was to start with the World Health Organization Model List of Essential Medicines-21st list, 2019 [13] and the WHO Model List of Essential Medicines for Children-7th list, 2019 [14] to extract all essential medicines that were indicated for the treatment of rare diseases. This exercise identified 26 medicines on the FDA, EMA, and/or China NMPA lists that were also on the WHO essential medicines lists; however, the
WHO indication was often not for a rare disease but a more common condition. Some key exceptions are medicines for treating hemophilia, cystic fibrosis, Marfan syndrome, Prader-Willi syndrome, myasthenia gravis, and sickle cell disease.

It is important to note that this collated list does not include any rare cancer drugs. Given the large number and the uniqueness, rare cancers deserve a separate list [15]. The European Joint Action on Rare Cancers initiated this work by conducting a survey for health professionals leading to the identification of 68 essential medicines for the treatment of pediatric malignancies according to best standards [16].

The core WG collated the initial list of medicinal products by eliminating duplicates and combining medicines that were ostensibly versions of a single drug therapy. While strict inclusion and exclusion criteria were not employed in compiling the RD drug list, all the entries were required to be approved by a major regulatory agency. In addition, all were recognized by the authors and the consultant group to have a reasonable risk/benefit ratio and to represent accepted treatments for the diseases under consideration. Drugs were excluded if they had orphan designation but lacked approval, if approval was withdrawn, or if the drug was known to have unacceptable side effects. Some medications in Table 1 are indicated for common disorders but are also treatments for rare subsets of the disorder (e.g., pediatric ulcerative colitis).

The goal of the RDTAWG for this first stage of work was identified as the creation of a list of RD medicines that, based on orphan designation and approval or marketing authorization, were efficacious, safe and having a significant impact on the quality and/or duration of life. In some cases, they could be considered standards of care based on widespread and long-term use; however, no attempt was made to categorize the drugs according to life-saving, curative, or beneficial properties.

Moreover, while it was desirable that the medicines on the list could be managed across a variety of countries at different stages of health system development, there was no detailed assessment on the basis of cost-effectiveness, complexity of management, or requirements for administration. Hence, unlike the WHO list of essential medicines, this list of RD drugs is not stratified nor prioritized on the basis of various criteria that could affect feasibility of adoption.

This list is intended to be the initial iteration of a "living document", to be revised and updated periodically. The list is not based on definitive criteria for inclusion nor is it the product of an expert consensus process. It is not intended to be comprehensive but is proposed to the rare disease community for consideration and uptake 
Table 1 List of essential medicinal products for rare diseases

\begin{tabular}{|c|c|c|c|c|}
\hline & Condition & Drug & Approvals & WHO \\
\hline \multicolumn{5}{|c|}{ Metabolic } \\
\hline & \multicolumn{4}{|l|}{ Aminoacid Disorders } \\
\hline & \multirow[t]{2}{*}{ Urea cycle disorders } & Benzoate and phenylacetate & FDA & \\
\hline & & Sodium phenylbutyrate & FDA, EMA & \\
\hline & $\mathrm{N}$-acetylglutamate synthetase deficiency & Carglumic acid & FDA, EMA & \\
\hline & Homocystinuria & Betaine & FDA, EMA & \\
\hline & Hyperphenylalaninemia & Sapropterin & FDA, EMA, NMPA & \\
\hline & Tetrahydrobiopterin deficiency & Sapropterin & FDA, EMA, NMPA & \\
\hline & Phenylketonuria & Pegvaliase & FDA, EMA & \\
\hline & Tyrosinemia type 1 & Nitisinone & FDA, EMA & \\
\hline & Alkaptonuria & Nitisinone & EMA & \\
\hline & \multicolumn{4}{|l|}{ Lysosomal Storage Diseases } \\
\hline & \multirow[t]{5}{*}{ Gaucher disease } & Miglustat & FDA, EMA, NMPA & \\
\hline & & Eliglustat & FDA, EMA & \\
\hline & & Velaglucerase alfa & FDA, EMA & \\
\hline & & Imiglucerase & FDA, EMA, NMPA & \\
\hline & & Taliglucerase & FDA, EMA & \\
\hline & \multirow[t]{3}{*}{ Fabry disease (alphagalactosidase A deficiency) } & Agalsidase beta & EMA, NMPA & \\
\hline & & Agalsidase alfa & EMA, NMPA & \\
\hline & & Migalastat & FDA, EMA & \\
\hline & $\begin{array}{l}\text { Lysosomal acid lipase deficiency, Wolman disease, Cholesteryl } \\
\text { ester storage disease }\end{array}$ & Sebelipase alfa & FDA, EMA & \\
\hline & Pompe disease & Alglucosidase alfa & FDA, EMA, NMPA & \\
\hline & Alpha mannosidosis & Velmanase alfa & EMA & \\
\hline & Mucopolysaccharidosis I (Iduronidase deficiency) & Laronidase & EMA, NMPA & \\
\hline & Hunter syndrome (Mucopolysaccharidosis II) & Idursulfase & FDA, EMA, NMPA & \\
\hline & Mucopolysaccharidosis IV (Morquio A syndrome) & Elosulfase alfa & FDA, EMA, NMPA & \\
\hline & Mucopolysaccharidosis VI (Maroteaux-Lamy syndrome) & Galsulfase & EMA & \\
\hline & Mucopolysaccharidosis VII (Sly syndrome) & Vestronidase alfa & FDA, EMA & \\
\hline & Neuronal ceroid lipofuscinosis type 2 & Cerliponase alfa & FDA, EMA & \\
\hline & \multirow[t]{3}{*}{ Nephropathic cystinosis } & Cysteamine & FDA, EMA & \\
\hline & & Cysteamine (enteric coated) & FDA, EMA & \\
\hline & & Cysteamine hydrochloride eyedrops & FDA, EMA & \\
\hline & \multicolumn{4}{|l|}{ Cholesterol, Lipid, Fatty Acid Disorders } \\
\hline & \multirow[t]{3}{*}{ Homozygous familial hypercholesterolemia } & Evolocumab & FDA, EMA, NMPA & \\
\hline & & Rosuvastatin calcium & FDA, NMPA & \\
\hline & & Lomitapide & FDA, EMA & \\
\hline & Cholesterol and bile acid synthesis defects & Cholic acid & FDA, EMA, NMPA & \\
\hline & Cerebrotendinous xanthomatosis & Chenodeoxycholic acid & EMA & \\
\hline & Familial chylomicronemia syndrome & Volanesorsen & EMA & \\
\hline & Congenital or hereditary chronic cholestasis & Tocofersolan & EMA & \\
\hline & \multicolumn{4}{|l|}{ Other Metabolic Disorders } \\
\hline & Pediatric onset hypophosphatasia & Asfotase alfa & FDA, EMA & \\
\hline & Hypophosphatemic rickets (x-Linked) & Burosumab-twza & FDA, EMA & \\
\hline & Hyperphosphatemia in renal failure & Calcium acetate & FDA & \\
\hline & Osteogenesis imperfecta & Alendronate & NMPA & \\
\hline & Scurvy & Ascorbic acid & FDA & WHO \\
\hline & \multirow[t]{2}{*}{ Metabolic acidosis } & Thiamine & EMA & WHO \\
\hline & & Trisodium citrate & EMA & \\
\hline & Genetic carnitine deficiency & Levocarnitine & FDA, NMPA & \\
\hline
\end{tabular}


Table 1 (continued)

\begin{tabular}{|c|c|c|c|c|}
\hline & Condition & Drug & Approvals & WHO \\
\hline & Fatty acid oxidation disorders & Triheptanoin & FDA & \\
\hline & Acyl Coenzyme A dehydrogenase deficiency & Riboflavin & EMA & WHO \\
\hline & Hereditary orotic aciduria & Uridine triacetate & FDA & \\
\hline & Prevention of uric acid nephrolithiasis & Potassium citrate & FDA & \\
\hline & Prevention of cystine nephrolithiasis (cystinuria) & Tiopronin & FDA & \\
\hline & Wilson disease & Penicillamine & NMPA & WHO \\
\hline & & Trientine $\mathrm{HCl}$ & FDA, EMA & \\
\hline & & Zinc acetate & FDA, EMA & \\
\hline & Cobalamin defects & Hydroxocobalamin & FDA & WHO \\
\hline \multicolumn{5}{|l|}{ Neurologic } \\
\hline & \multicolumn{4}{|l|}{ General } \\
\hline & \multirow[t]{3}{*}{ Transthyretin amyloidosis } & Inotersen & FDA, EMA & \\
\hline & & Tafamidis & FDA, EMA & \\
\hline & & Patisiran sodium & FDA, EMA & \\
\hline & \multirow[t]{3}{*}{ Multiple Sclerosis } & Teriflunomide & EMA, NMPA & \\
\hline & & Fingolimod $\mathrm{HCl}$ & EMA, NMPA & \\
\hline & & Siponimod & NMPA & \\
\hline & \multirow[t]{4}{*}{ Parkinson Disease (Young and Early-onset) } & Rasagiline & EMA, NMPA & \\
\hline & & Selegiline & FDA, EMA, NMPA & \\
\hline & & Pramipexole & EMA, NMPA & \\
\hline & & Carbidopa/Levodopa & FDA, EMA & WHO \\
\hline & \multirow[t]{2}{*}{ Narcolepsy with cataplexy } & Pitolisant & FDA, EMA & \\
\hline & & Sodium oxybate & FDA, EMA & \\
\hline & \multirow[t]{2}{*}{ Huntington Disease } & Deutetrabenazine & NMPA & \\
\hline & & Tetrabenazine & FDA, EMA & \\
\hline & Dystonia, Spasticity & Baclofen & FDA & \\
\hline & Tuberous Sclerosis Complex & Everolimus & FDA, EMA & \\
\hline & Spina bifida (prevention) & Folic acid & EMA & WHO \\
\hline & Biotinidase deficiency & Biotin & NMPA & \\
\hline & \multicolumn{4}{|l|}{ Epilepsy } \\
\hline & Infantile spasms & Vigabatrin & FDA, EMA & \\
\hline & \multirow[t]{2}{*}{ Lennox-Gastaut syndrome } & Rufinamide & FDA, EMA & \\
\hline & & Cannabidiol & FDA, EMA & \\
\hline & Severe myoclonic epilepsy in infancy (Dravet syndrome) & Stiripentol & FDA, EMA & \\
\hline & Status epilepticus & Midazolam & FDA & WHO \\
\hline & Juvenile myoclonic epilepsy, Generalized epilepsy & Levetiracetam & EMA & \\
\hline & \multirow[t]{3}{*}{ Complex and rare disease epilepsy } & Clobazam & FDA & \\
\hline & & Lamotrigine & FDA & WHO \\
\hline & & Topiramate & FDA & \\
\hline & \multicolumn{4}{|l|}{ Neuromuscular Diseases } \\
\hline & \multirow[t]{3}{*}{ Amyotrophic lateral sclerosis } & Gabapentin & FDA & \\
\hline & & Riluzole & FDA, EMA, NMPA & \\
\hline & & Radicava & NMPA & \\
\hline & Myasthenia gravis & Pyridostigmine Bromide & NMPA & WHO \\
\hline & Lambert-Eaton myasthenic syndrome & Amifampridine & EMA & \\
\hline & Non-dystrophic myotonic disorders & Mexiletine hol & EMA & \\
\hline & 5q Spinal Muscular Atrophy & Nusinersen sodium & FDA, EMA, NMPA & \\
\hline \multicolumn{5}{|l|}{ Hematologic } \\
\hline & \multicolumn{4}{|l|}{ Coagulation Defects } \\
\hline
\end{tabular}


Table 1 (continued)

\begin{tabular}{|c|c|c|c|c|}
\hline & Condition & Drug & Approvals & WHO \\
\hline \multirow{12}{*}{\multicolumn{2}{|c|}{ Hemophilia A (Factor VIII deficiency) }} & Octocog alpha & EMA & \\
\hline & & Rurioctocog alfa pegol & EMA & \\
\hline & & Lonoctocog alfa & EMA & \\
\hline & & Emicizumab & FDA, EMA, NMPA & \\
\hline & & Damoctocog alfa pegol & EMA & \\
\hline & & Turoctocog alpha & EMA & \\
\hline & & Simoctocog alfa & EMA & \\
\hline & & Moroctocog alpha & EMA & \\
\hline & & Desmopressin acetate & FDA, EMA & WHO \\
\hline & & Recombinant Factor VIII & EMA, NMPA & \\
\hline & & Efmoroctocog alfa & EMA & \\
\hline & & Factor VIII/ von Willebrand factor & EMA & \\
\hline \multicolumn{2}{|r|}{ von Willebrand disease } & Vonicog alfa & EMA & \\
\hline \multirow{7}{*}{\multicolumn{2}{|c|}{ Hemophilia B (Factor IX deficiency) }} & Eftrenonacog alfa & EMA & \\
\hline & & Albutrepenonacog alfa & EMA & \\
\hline & & Nonacog alpha & EMA & \\
\hline & & Human coagulation factor IX & EMA & WHO \\
\hline & & Nonacog beta pegol & EMA & \\
\hline & & Nonacog gamma & EMA & \\
\hline & & Recombinant Factor IX & EMA, NMPA & \\
\hline \multirow{2}{*}{\multicolumn{2}{|c|}{ Hemophilia (Factor VII deficiency) }} & Eptacog alpha (activated) & EMA & \\
\hline & & Recombinant Factor VIla & EMA & \\
\hline & Factor $\mathrm{X}$ deficiency & Human coagulation factor $X$ & EMA & \\
\hline & Factor XIII A-subunit deficiency & Catridecacog & EMA & \\
\hline & Protein C deficiency & Human protein c & EMA & \\
\hline \multicolumn{5}{|c|}{ Anemias } \\
\hline & Sickle cell anemia & Hydroxyurea & FDA & \\
\hline & Anemia of end-stage renal disease & Epoetin alfa & FDA & WHO \\
\hline & Idiopathic thrombocytopenic purpura, Aplastic anemia & Eltrombopag & FDA, EMA & \\
\hline & Beta thalassemia major & Deferasirox & FDA, EMA, NMPA & \\
\hline \multicolumn{5}{|c|}{ Other Hematologic Disorders } \\
\hline & Congenital and acquired methemoglobinemia & Methylene blue injection & FDA & \\
\hline & Acute intermittent porphyria & Hemin & FDA & \\
\hline & Erythropoietic protoporphyria & Afamelanotide & FDA, EMA & \\
\hline & Multicentric Castleman's disease & Siltuximab & FDA, EMA & \\
\hline & Essential thrombocythemia & Anagrelide hydrochloride & FDA, EMA & \\
\hline & Paroxysmal nocturnal hemoglobinuria & Ravulizumab & FDA, EMA & \\
\hline & Severe congenital neutropenia & Macapegfilgrastim & NMPA & \\
\hline & \multirow[t]{2}{*}{ Conditioning for hematopoietic stem cell transplant } & Busulfan & FDA, EMA & \\
\hline & & Thiotepa & FDA, EMA & \\
\hline & Iron overload & Deferiprone & FDA, EMA & \\
\hline & Acquired thrombotic thrombocytopenic purpura & Caplacizumab & FDA, EMA & \\
\hline & Immune (idiopathic) thrombocytopenic purpura & Romiplostim & FDA, EMA & \\
\hline & \multirow[t]{2}{*}{ Polycythemia vera } & Ropeginterferon alfa-2b & EMA & \\
\hline & & Ruxolitinib & FDA, EMA & \\
\hline & Agammaglobulinemia & Immunoglobulin infusion & NMPA & \\
\hline \multicolumn{5}{|l|}{ Inflammatory } \\
\hline & \multicolumn{4}{|l|}{ Rheumatoid Arthritis } \\
\hline & Juvenile rheumatoid arthritis & Methotrexate & FDA, EMA & WHO \\
\hline
\end{tabular}


Table 1 (continued)

\begin{tabular}{|c|c|c|c|c|}
\hline & Condition & Drug & Approvals & WHO \\
\hline & & Etanercept & FDA, EMA & \\
\hline & & Methylprednisolone & EMA & WHO \\
\hline & & Adalimumab & FDA & \\
\hline & & Infliximab & FDA & \\
\hline & & Tocilizumab & FDA, EMA & \\
\hline & & Abatacept & EMA & \\
\hline & & Golimumab & FDA, EMA & \\
\hline & \multicolumn{4}{|l|}{ Gastrointestinal Inflammation } \\
\hline & \multirow[t]{2}{*}{ Pediatric Crohn's disease } & Adalimumab & FDA & \\
\hline & & Infliximab & FDA & \\
\hline & \multirow[t]{4}{*}{ Pediatric ulcerative colitis } & Mesalamine & FDA & \\
\hline & & 5-aminosalicylic acid & FDA & \\
\hline & & Adalimumab & FDA & \\
\hline & & Infliximab & FDA & \\
\hline & Primary biliary cholangitis & Obeticholic acid & FDA, EMA & \\
\hline & Hereditary chronic cholestasis & Tocofersolan & EMA & \\
\hline & \multicolumn{4}{|l|}{ Angioedema } \\
\hline & \multirow[t]{5}{*}{ Hereditary angioedema } & C1 inhibitor(human) & EMA & \\
\hline & & Icatibant acetate & FDA, EMA & \\
\hline & & Lanadelumab & FDA, EMA, NMPA & \\
\hline & & Danazol & NMPA & \\
\hline & & Tranexamic acid & FDA, NMPA & WHO \\
\hline & \multirow[t]{2}{*}{ Angioedema due to $\mathrm{C} 1$ esterase inhibitor deficiency } & C1-esterase-inhibitor, human & FDA & \\
\hline & & Conestat alfa & EMA & \\
\hline & \multicolumn{4}{|l|}{ Other Inflammatory Disorders } \\
\hline & Multiple sclerosis, Behcet's disease, Familial Mediterranean fever & Colchicine & FDA, NMPA & \\
\hline & $\begin{array}{l}\text { Dermatomyositis, Atypical hemolytic uremic syndrome, Neuromy- } \\
\text { elitis Optica, Paroxysmal nocturnal hemoglobinuria, Myasthenia } \\
\text { gravis }\end{array}$ & Eculizumab & FDA, EMA, NMPA & \\
\hline & $\begin{array}{l}\text { Anti-neutrophil vasculitis, Wegener's granulomatosis, Churg- } \\
\text { Strauss Syndrome }\end{array}$ & Rituximab & FDA & WHO \\
\hline & Familial Mediterranean fever, Cryopyrin fevers & Canakinumab & FDA, EMA, NMPA & \\
\hline & Still's disease, Systemic juvenile arthritis & IL-1 Receptor antagonist anakinra & FDA, EMA & \\
\hline & Neurotrophic keratitis & Cenegermin & FDA, EMA & \\
\hline & Vernal keratoconjunctivitis & Ciclosporin & EMA & WHO \\
\hline & Non-infectious uveitis & Dexamethasone & FDA, EMA & WHO \\
\hline & Cryopyrin-associated periodic syndromes & Rilonacept & FDA, EMA & \\
\hline \multicolumn{5}{|l|}{ Endocrine } \\
\hline & Growth hormone deficiency in children & Somatropin for injection & FDA, EMA & \\
\hline & \multirow[t]{4}{*}{ Acromegaly } & Octreotide & FDA & \\
\hline & & Lanreotide & FDA & \\
\hline & & Pegvisomant & FDA, EMA & \\
\hline & & Pasireotide & FDA, EMA & \\
\hline & \multirow[t]{2}{*}{ Endogenous Cushing's syndrome } & Osilodrostat & FDA, EMA & \\
\hline & & Ketoconazole & EMA & \\
\hline & Adrenal insufficiency & Hydrocortisone & FDA, EMA, NMPA & WHO \\
\hline & \multirow[t]{2}{*}{ Idiopathic Hypogonadotropic Hypogonadism } & Human chorionic gonadotropin & EMA, NMPA & \\
\hline & & Gonadotropin-releasing hormone & EMA, NMPA & \\
\hline & Primary insulin-like growth factor-1 deficiency & Mecasermin & FDA, EMA & \\
\hline & Paget's disease (osteitis deformans) & Calcitonin-human for injection & FDA & \\
\hline
\end{tabular}


Table 1 (continued)

\begin{tabular}{|c|c|c|c|c|}
\hline & Condition & Drug & Approvals & WHO \\
\hline & Hypoparathyroidism & Parathyroid hormone & FDA, EMA & \\
\hline & Non-24-h sleep-wake disorder & Tasimelteon & FDA, EMA & \\
\hline & Leptin deficiency in lipodystrophy patients & Metreleptin & FDA, EMA & \\
\hline & Familial partial lipodystrophy & Metreleptin & EMA & \\
\hline \multicolumn{5}{|l|}{ Pulmonary } \\
\hline & \multirow[t]{10}{*}{ Pulmonary arterial hypertension } & Macitentan & FDA, EMA, NMPA & \\
\hline & & Tadalafil & FDA, EMA & \\
\hline & & Ambrisentan & FDA, EMA, NMPA & \\
\hline & & Nitric oxide & FDA, EMA & \\
\hline & & Sildenafil & EMA, NMPA & \\
\hline & & Bosentan monohydrate & FDA, EMA, NMPA & \\
\hline & & Selexipag & FDA, EMA, NMPA & \\
\hline & & Iloprost & FDA, EMA, NMPA & \\
\hline & & Parenteral treprostinil & FDA, EMA, NMPA & \\
\hline & & Riociguat & FDA, EMA, NMPA & \\
\hline & \multirow[t]{8}{*}{ Cystic fibrosis } & Mannitol & FDA, EMA & WHO \\
\hline & & Ivacaftor & FDA, EMA & \\
\hline & & Tezacaftor/ivacaftor & FDA, EMA & \\
\hline & & Tobramycin & FDA, EMA & \\
\hline & & Aztreonam & FDA, EMA & \\
\hline & & Colistimethate sodium & EMA & \\
\hline & & Lumacaftor / ivacaftor & FDA, EMA & \\
\hline & & Levofloxacin & EMA & WHO \\
\hline & \multirow[t]{2}{*}{ Idiopathic Pulmonary Fibrosis } & Pirfenidone & FDA, EMA & \\
\hline & & Nintedanib & FDA, EMA, NMPA & \\
\hline & Primary apnea of premature newborns & Caffeine citrate & FDA, EMA & WHO \\
\hline & Lymphangioleiomyomatosis, Tuberous sclerosis & Sirolimus & FDA, EMA & \\
\hline \multicolumn{5}{|l|}{ Immunologic } \\
\hline & $\begin{array}{l}\text { Severe combined immunodeficiency, Adenosine deaminase } \\
\text { deficiency }\end{array}$ & Pegademase bovine & FDA & \\
\hline & & CD34 + cells transduced with ADA cDNA & EMA & \\
\hline & Chronic granulomatous disease & Interferon gamma 1-b & FDA & \\
\hline \multicolumn{5}{|l|}{ Miscellaneous } \\
\hline & Mastocytosis & Cromolyn sodium & FDA & \\
\hline & Ventricular tachycardia & Amiodarone & FDA & WHO \\
\hline & Limbal stem cell deficiency & Autologous human corneal stem cells & EMA & \\
\hline & Inherited retinal dystrophy & Voretigene neparvovec & FDA, EMA & \\
\hline & Short bowel syndrome & Teduglutide & FDA, EMA & \\
\hline & Hepatic veno-occlusive disease, Sinusoidal obstruction & Defibrotide & FDA, EMA & \\
\hline & Autosomal dominant polycystic kidney disease & Tolvaptan & FDA, EMA & \\
\hline & Patent ductus arteriosus & Ibuprofen & FDA, EMA & WHO \\
\hline & Anthracycline extravasation & Dexrazoxane & FDA, EMA & \\
\hline
\end{tabular}

List of 204 essential medicinal products for rare diseases with marketing authorization extracted from the FDA database and/or EMA database and/or Chinas's Rare Diseases Catalog

as well as a starting point or guide for jurisdictions to set policies on provision of rare disease medicines to their populations.

The compiled list was sent to a group of rare disease specialists (as listed in Acknowledgments) with instructions to review those medications within their area(s) of expertise and, specifically, to eliminate duplicate or redundant medicines, remove drugs considered inappropriate or ineffective, add other drugs that should be on the list, and provide comments as appropriate. 
Feedback was discussed by all members of the core RDTAWG to arrive at a consensus whether to accept recommendations, or not, to arrive at a final collated list.

\section{Results}

The Table 1 presents the current working list of essential rare disease medicines with different versions of a medication listed separately where appropriate. The list is organized into seven disease categories: metabolic, neurologic, hematologic, anti-inflammatory, endocrine, pulmonary, and immunologic, plus a miscellaneous category. Moreover, some diseases cluster together in groups (e.g. Wolman disease and Cholesteryl ester storage disease cluster in the group of Lysosomal acid lipase deficiency). However, in identifying the total number of diseases, these diseases are counted individually and not as a single cluster. Within each category, drugs are listed by subgroupings and specific conditions, with multiple indications where appropriate. The third column gives the agencies that approved the drug; the fourth column notes drugs that are on the WHO Model List of Essential Medicines (21st edition). Some of the drugs have indications beyond those listed in the table. The drugs are not coded in terms of priority, therapeutic strength or equivalence, need for specialized diagnosis or care, or any restrictions (cf. WHO Model List of Essential Medicines). The greatest number of drugs is in the metabolic disease category, but various neurological diseases are extensively represented.

Table 2 provides a summary on the total number of listed diseases, the number of diseases per category, the number of total medicinal products, the number of drugs and biologics, the number of medicinal products per disease category, the number of medicinal products treating more than one disease, and the number of medicinal products approved in each jurisdiction. A total of 134 diseases are listed; among these, the largest category consists of metabolic conditions $(n=40)$, followed by hematologic conditions $(n=24)$, inflammatory $(n=23)$, neurologic $(n=20)$, endocrine $(n=11)$, miscellaneous $(\mathrm{n}=9)$, pulmonary $(\mathrm{n}=5)$ and immunologic $(\mathrm{n}=2)$.

Additionally, as noted in Table 2, the list includes 204 drugs, of which 125 are chemical drugs and 79 are classified as biologics, which also includes recombinant proteins, polyclonal and monoclonal antibodies, and cell and nucleic acid therapies. There are six drugs that treat more than one disease (Additional file 1).

\section{Discussion}

Individuals with rare diseases encounter many challenges along the path to appropriate care and treatment. The first obstacle for many is obtaining an accurate diagnosis, which often takes more than 5 years [17]. For many, the
Table 2 Number of diseases and medicinal products per category

\begin{tabular}{|c|c|}
\hline & Number \\
\hline \multicolumn{2}{|l|}{ Diseases } \\
\hline Total & 134 \\
\hline Metabolic/inborn errors & 40 \\
\hline Neurologic & 20 \\
\hline Hematologic & 24 \\
\hline Inflammatory & 23 \\
\hline Endocrine & 11 \\
\hline Pulmonary & 5 \\
\hline Immunologic & 2 \\
\hline Miscellaneous & 9 \\
\hline \multicolumn{2}{|l|}{ Medicinal products } \\
\hline Total medicinal products (MP) & 204 \\
\hline Drugs & 125 \\
\hline Biologics & 79 \\
\hline MP for metobolic diseases & 51 \\
\hline MP for neurologic diseases & 34 \\
\hline MP for hematologic diseases & 43 \\
\hline MP for inflammatory diseases & 28 \\
\hline MP for endocrine diseases & 15 \\
\hline MP for pulmonary diseases & 22 \\
\hline MP for immunologic diseases & 3 \\
\hline MP for miscellaneous & 9 \\
\hline MP used to treat multiple diseases & 6 \\
\hline MP approved by the FDA & 139 \\
\hline MP approved by the EMA & 160 \\
\hline MP approved by the NMPA & 51 \\
\hline MP on the WHO list of essential medicines (2019) & 25 \\
\hline
\end{tabular}

Summary of the total number of listed diseases, the number of diseases per category, the number of total medicinal products, the number of drugs and biologics, the number of medicinal products per disease category, the number of medicinal products treating multiple diseases, and the number of medicinal products approved in each jurisdiction

next hurdle involves finding expert care and treatment, which can vary depending upon many factors including geographic location and socioeconomic status. In fact, researchers have noted profound disparities across the globe in access to rare disease medicines, with significant impact on health outcomes and quality of life $[18,19]$. In 2006, Stolk et al. [20] called for inclusion of RD drugs as essential medicines, but this has not occurred.

Many of the drugs in our RD drug list are not included in the WHO Model List of Essential Medicines.

We note that not all of the drugs on our list are approved across all jurisdictions, and a few with regulatory approval and/or marketing authorization are not indicated for the specified rare disease(s), even if they are recognized as a standard of care or appropriate. Based upon such a lack of indication, some health systems may choose to deny reimbursement even if the 
drug is inexpensive, genericized and in distribution. This problem affects patients in high-income as well as lowand-middle income countries. Therefore, it is important to take a broader contextual approach to understand the challenges rare disease patients are facing and address them collectively and systematically. Moreover, some of the drugs listed in Table 1 are applicable to only a fraction of the patients who have the associated disease. Ivacaftor for certain genetic subtypes of cystic fibrosis is one example.

Approximately one-third of all persons worldwide, including those in low-income but also middle-income countries, do not have access to essential medicines, specifically drugs, vaccines, and diagnostics for communicable, noncommunicable, social-behavioral illnesses, and emerging environmentally induced diseases [1]. The cause of the problem, like the cause of the diseases, is multifactorial and requires not only multidisciplinary and multisectoral approaches but integrated, holistic innovative solutions. Barriers at the individual level include the lack of health literacy, awareness of therapies, and advocacy capacity. Healthcare professionals similarly may lack awareness of appropriate medicines, knowledge to use effectively, and capacity to advocate for access. Major impediments at the systems level include lack of lower cost alternatives (generics and biosimilars) as well as the lack of regulatory, clinical and infrastructure capacity to make complex innovative therapies available and to deliver them to patients [21]. For example, depending of the type of therapies (chemical drug/biologic/gene or cell therapy), countries or jurisdictions may have to consider the feasibility of establishing and maintaining manufacturing and delivery chains relative to their local capacities. In addition, while nations may be criticized for limited national commitment to healthcare and insufficient investment in universal health coverage, criticism may also be levied on industry for the lack of transparency and high pricing that compromise a nation's ability to deliver optimal healthcare; this critique is punctuated by the WHO resolution on disclosure of drug prices [22].

Many of the aforementioned challenges (especially regulatory expertise and clinical capacity) have a disproportionate impact on rare disease drugs and patients, but there are additional barriers. Some are grounded in "high evidential uncertainty" in extending clinical trial data to real-world outcomes. This is highly problematic in countries that apply "traditional" health technology assessment (HTA) or value-based assessment (VBA) methodology to RD therapies compared to those jurisdictions that use supplemental processes with greater flexibilities that treat RD treatments differently [23].

How could this list of RD medicines be used? A potential pathway is one based on EMA's EU-Medicines4all
(EUM4all) procedure. EMA established EUM4all to provide expert reviews on benefits and risks of medicines that would be used outside the EU, with emphasis on LMICs [24]. Subsequent analysis found that 138 regulatory approvals had been granted in 90 different countries worldwide for six medicines based on EUM4all opinions, with acknowledged great public health impact.

The EUM4all initiative dealt with a broad range of medicines with high impact in LMICs, but we propose that the procedure could profitably be applied to RD medicines. This paper is intended to elicit suggestions and call for collaborations on how to modify, disseminate, and use the list of medicines in the Table 1. Specifically, the RDTAWG seeks input from RD advocacy groups, healthcare providers, pharmaceutical companies, and government agencies. Subsequent actions include a conference to bring together key stakeholders to elaborate on the list, identify barriers and opportunities for application and collaborate on next steps. The ultimate goal is to enhance access to appropriate medicines for all rare disease patients throughout the world.

\section{Conclusions}

The limited number of approved therapeutic options, combined with the unavailability of existing treatments, significantly impair the life of rare disease patients in LMICs. While many countries have recently developed policies and regulations for rare diseases and orphan drugs, access to treatment remains variable among LMICs. With the vision of leaving no one behind, the IRDiRC RDTAWG used the FDA, EMA and China NMPA databases to extract approved drugs and create the first list of 204 essential medicinal products for rare diseases. The list was organized into seven disease categories, excluding rare cancers and rare infectious diseases. The ultimate goal of this list is to further stimulate interactions among patient organizations, health care providers, industry and government agencies to improve standards of care for rare diseases by promoting access to treatments.

\section{Abbreviations \\ IRDiRC: International Rare Diseases Research Consortium; RDTAWG: Rare Disease Treatment Access Working Group; RD: Rare Diseases; LMICs: Low-and- Middle-Income Countries; OMP: Orphan Medicinal Products; FDA: US Food and Drug Administration; EMA: European Medicines Agency; WHO: World Health Organization; EU: European Union; NMPA: National Medical Products Administration of China; HTA: Health Technology Assessment; VBA: Value Based Assessment.}

\section{Supplementary Information}

The online version contains supplementary material available at https://doi. org/10.1186/s13023-021-01923-0. 
Additional file 1. Used to complement the information provided in Table 2. Individual diseases (and not cluster of diseases) are listed by alphabetic order for each category. Medicinal products are filtered by alphabetic order and listed for each disease category. The 6 medicinal products used to treat multiple diseases and the 79 biologics are listed.

\section{Acknowledgements}

Core group members of the RDTAWG include WG, DWR, SG, SW, VH, RY, GZ In addition, we would like to thank Rachel Bishop (National Eye Institute, $\mathrm{NIH}$, USA), Marc Dooms (UZ Leuven, Belgium), Katharina Hohenfellner (RoMed Klinikum Rosenheim, Germany), Bela Melegh (University of Pecs, Hungary), Filippo Pinto e Vairo (Mayo Clinic, USA) and Manuel Posada (Instituto de Salud Carlos III, Spain) for their review of the list of essential medicinal products for rare diseases.

\section{Authors' contributions}

The RDTAWG was led by WG and DWR. WG extracted all the drugs with orphan designations and FDA approval, and initiated the redaction of the manuscript. SG revised the list of drugs extracted by WG. VH used the Orphanet and EMA databases to collate medicinal products for rare diseases that have European Union marketing authorizations. RY consulted China's Rare Diseases Catalog and China's first three Lists of Novel Drugs Approved in Other Jurisdictions with Urgent Clinical Need to identify the drugs approved for the treatment of recognized rare conditions. WG, DWR, SG, VH, RY, GZ revised the list of medicinal products, edited the manuscript and validated its final version. All authors read and approved the final manuscript.

\section{Funding}

The IRDiRC Scientific Secretariat is funded by the European Union through the European Joint Programme on Rare Disease under the European Union's Horizon 2020 research and innovation programme Grant Agreement $N^{\circ} 825575$. The Scientific Secretariat is hosted at the French Institute of Health and Medical Research (INSERM) in Paris, France. This work was supported in part by the Intramural Research Program of the National Human Genome Research Institute.

\section{Availability of data and materials}

The datasets analyzed as sources for the lists are available from the corresponding author upon request.

\section{Declarations}

\section{Ethics approval and consent to participate}

The study does not involve human participants, human data or human tissue. No ethics approval and consent were required.

\section{Consent for publication}

The study does not contain any individual person's data.

\section{Competing interests}

The authors declare that the research was conducted in the absence of any commercial or financial relationships that could be construed as a potential conflict of interest.

\section{Author details}

${ }^{1}$ National Human Genome Research Institute, NIH, Bethesda, USA. ${ }^{2}$ Canadian Organization for Rare Disorders, Toronto, Canada. ${ }^{3}$ EURORDIS-Rare Diseases Europe, Paris, France. ${ }^{4}$ China Alliance for Rare Diseases, Geneva, Switzerland. ${ }^{5}$ IRDiRC Scientific Secretariat, Inserm, Paris, France. ${ }^{6}$ National Center for Advancing Translational Sciences, NIH, Bethesda, USA.

Received: 16 March 2021 Accepted: 20 June 2021

Published online: 13 July 2021

\section{References}

1. Stevens $\mathrm{H}$, Huys I. Innovative approaches to increase access to medicines in developing countries. Front Med. 2017;4:218.

2. Detiček A, Locatelli I, Kos M. Patient access to medicines for rare diseases in European countries. Value Health. 2018;21:553-60.

3. Chan AYL, Chan VKY, Olsson S, Fan M, Jit M, Gong M, et al. Access and unmet needs of orphan drugs in 194 countries and 6 areas: a global policy review with content analysis. Value Health. 2020;23:1580-91.

4. Kishore SP, Kolappa K, Jarvis JD, Park PH, Belt R, Balasubramaniam T, et al. Overcoming obstacles to enable access to medicines for noncommunicable diseases in poor countries. Health Aff. 2015;34:1569-77.

5. Search Orphan Drug Designations and Approvals [Internet]. U.S. FOOD \& DRUG Administration. [cited 2021 Jan 29]. https://www.accessdata.fda. gov/scripts/opdlisting/oopd/.

6. Lists of medicinal products for rare diseases in Europe [Internet]. Orphanet; 2020. https://www.orpha.net/orphacom/cahiers/docs/GB/list_ of_orphan_drugs_in_europe.pdf.

7. Medicines Search [Internet]. European Medicines Agency. [cited 2021 Jan 29]. https://www.ema.europa.eu/en/medicines/ema_group_types/ema_ orphan.

8. China National Rare Disease Registry [Internet]. [cited 2021 Jan 29]. https://www.nrdrs.org.cn/app/rare/index.html.

9. Drug Evaluation Center of the State Drug Administration [Internet]. [cited 2021 Jan 29]. http://www.cde.org.cn/.

10. Notice on the issuance of the first batch of clinically urgently needed overseas new drugs. [Internet]. [cited 2021 May 7]. http://www.cde.org. $\mathrm{cn} /$ news.do?method=largelnfo\&id=c059310700b4b383.

11. Notice on the issuance of the second batch of clinically urgently needed overseas new drugs. [Internet]. [cited 2021 May 7]. http://www.cde.org. $\mathrm{cn} /$ news.do?method=largelnfo\&id=8ed064bd7e51fe68.

12. Notice on the issuance of the third batch of clinically urgently needed overseas new drugs. [Internet]. [cited 2021 May 7]. http://www.cde.org. $\mathrm{cn} /$ news.do? method=largelnfo\&id $=5$ ed6430be031 fc66.

13. World Health Organization Model List of Essential Medicines - 21st List, 2019 [Internet]. World Health Organization. [cited 2021 Jan 29]. https:// www.who.int/publications-detail-redirect/WHOMVPEMPIAU2019.06.

14. World Health Organization Model List of Essential Medicines for Children, 7th List, 2019 [Internet]. World Health Organization; 2019. https://apps. who.int/iris/bitstream/handle/10665/325772/WHO-MVP-EMP-IAU-2019. 07-eng.pdf? sequence $=1$ \&isAllowed=y.

15. Prades J, Weinman A, Le Cam Y, Trama A, Frezza AM, Borras JM. Priorities on rare cancers' policy in National Cancer Control Plans (NCCPS): a review conducted within the framework of EU-JARC Joint-Action. J Cancer Policy. 2020;24:100222.

16. D9.1 Report summarising the results of the survey on accessibility of standard treatment and recommendations to Member States and Europe to overcome bottlenecks.pdf [Internet]. [cited 2021 May 7]. https://www. jointactionrarecancers.eu/images/work-packages/wp9/D9.1\%20Rep ort\%20summarising\%20the\%20results\%20of\%20the\%20survey\%20on\% 20accessibility\%20of\%20standard\%20treatment\%20and\%20recommen dations\%20to\%20Member\%20States\%20and\%20Europe\%20to\%20ove rcome\%20bottlenecks.pdf

17. Gahl WA, Wise AL, Ashley EA. The undiagnosed diseases network of the National Institutes of Health: a national extension. JAMA. 2015;314:1797.

18. Gammie T, Lu CY, Babar ZU-D. Access to Orphan drugs: a comprehensive review of legislations, regulations and policies in 35 countries. PLOS ONE. 2015;10:e0140002.

19. Czech M, Baran-Kooiker A, Atikeler K, Demirtshyan M, Gaitova K, Holownia-Voloskova M, et al. A review of rare disease policies and orphan drug reimbursement systems in 12 Eurasian countries. Front Public Health. 2020;7:416.

20. Stolk P. Rare essentials drugs for rare diseases as essential medicines. Bull World Health Organ. 2006;84:745-51.

21. Alsairi R. Access to medicine in developing countries. Am J Med Med Sci. 2017;7:271-6.

22. Fletcher ER. World Health Assembly Approves Milestone Resolution On Price Transparency [Internet]. Health Policy Watch; 2019 May. https://healt 
hpolicy-watch.news/world-health-assembly-approves-milestone-resol ution-on-price-transparency/.

23. Nicod E, Whittal A, Drummond M, Facey K. Are supplemental appraisal/ reimbursement processes needed for rare disease treatments? An international comparison of country approaches. Orphanet J Rare Dis. 2020;15:189.

24. Cavaller Bellaubi M, Harvey Allchurch M, Lagalice C, Saint-Raymond A. The European Medicines Agency facilitates access to medicines in lowand middle-income countries. Expert Rev Clin Pharmacol. 2020;13:321-5.

\section{Publisher's Note}

Springer Nature remains neutral with regard to jurisdictional claims in published maps and institutional affiliations.

Disclaimer The findings and recommendations in this article are those of the contributors, who participated based on their individual expertise and are responsible for the contents, and do not necessarily represent the views of the members of the International Rare Diseases Research Consortium (IRDiRC) nor any employers of the contributors.
Ready to submit your research? Choose BMC and benefit from:

- fast, convenient online submission

- thorough peer review by experienced researchers in your field

- rapid publication on acceptance

- support for research data, including large and complex data types

- gold Open Access which fosters wider collaboration and increased citations

- maximum visibility for your research: over $100 \mathrm{M}$ website views per year

At BMC, research is always in progress.

Learn more biomedcentral.com/submissions 